\title{
Avaliação de dano de Sitophilus zeamais, Oryzaephilus surinamensis e Laemophloeus minutus em grãos de arroz armazenados
}

\author{
Carlos E. Copatti', Roberta K. Marcon² \& Monaliza B. Machado²
}

RESUMO

Objetivou-se com o presente trabalho avaliar o efeito de predação de inseto praga primário, Sitophilus zeamais, e secundários, Oriza ephilus surinamensis e Laemophloeus minutus em grãos de arroz armazenados. As populações foram expostas a condições controladas de temperatura e fotoperíodo (20, 25 e $30^{\circ} \mathrm{C}, 14 \mathrm{~h}$ de fotofase) em cinco diferentes densidades de infestação (10, 20, 40, 80 e 120 indivíduos). Os tratamentos foram: S. zeamais isolado; O. surinamensis isolado; L. minutus isolado; S. zeamais + O. surinamensis e; $S$. zeamais + L. minutus. As análises de danos dos grãos de arroz ocorreram com 7 e 14 dias após o início dos experimentos separando os insetos praga dos grãos. As maiores perdas ocorreram com o aumento de temperatura e densidade de infestação; a coexistência $S$. zeamais $+L$. minutus apresentou os maiores efeitos de predação. Conclui-se que temperaturas mais baixas e monitoramento e controle de infestação de insetos praga primários e secundários reduzem a perda de grãos de arroz armazenados.

Palavras-chave: insetos praga, coexistência, temperatura, densidade de infestação

\section{Evaluation of damage by Sitophilus zeamais, Orizaephilus surinamensis and Laemophloeus minutus in stored rice grain}

\begin{abstract}
This study aimed to evaluate the predatory effects of the primary insect pests Sitophilus zeamais and the secondary insect pests Orizaephilus surinamensis and Laemophloeus minutus in stored rice grains. The insect populations were kept under controlled conditions of temperature and photoperiod $\left(20,25\right.$ and $30{ }^{\circ} \mathrm{C}, 14$ $\mathrm{h}$ photophase) in five different infestation densities (0,10,20,40,80 and 120 individuals). The treatments were: S. zeamais isolated; O. surinamensis isolated; L. minutus isolated; S. zeamais + O. surinamensis and; $S$. zeamais + L. minutus. Analysis of rice grain damage occurred at 7 and 14 days after the beginning of the experiments, separating the insect pests from the grains. The largest losses occurred with increasing temperature and infestation density and the coexistence of $S$. zeamais $+L$. minutus produced the greatest predatory effects. It is concluded that lower temperatures as well as monitoring and control of infestation of primary and secondary insect pests reduces the loss of rice grains stored.
\end{abstract}

Key words: insect pests, coexistence, temperature, infestation density 


\section{INTRODUÇ̃̃o}

O arroz (Oryza sativa L.) se destaca por ser um dos cereais mais cultivados do mundo, sendo base da alimentação de boa parte da população mundial (Copatti et al., 2009) além de sujeito a perdas quantitativas e qualitativas de acordo com as condições de armazenamento e presença de insetos praga. Segundo Ribeiro et al. (2012), a resistência do arroz a pragas de grãos armazenados é um caráter bastante influenciável pelo ambiente.

Geralmente, insetos apresentam elevadas densidades populacionais e diversidade, além de grande variedade de respostas à qualidade e à quantidade de recursos disponíveis (Copatti \& Gasparetto, 2012). Entre os insetos que se destacam pela ação direta aos grãos de arroz, ou seja, pela capacidade de danificar os grãos ainda sadios, se encontra Sitophilus zeamais (Coleoptera: Curculionidae) (Mots. 1855).

Sitophilus zeamais é uma praga primária considerada de maior importância para os grãos armazenados no Brasil por apresentar infestação cruzada, ser praga de profundidade, ter elevado potencial biótico e tanto na fase larval quanto na fase adulta, causar danos aos grãos (Silveira et al., 2006).

Já o Laemophloeus minutus (Coleoptera: Curculidae) (Oliv. 1791) e Orizaephilus surinamensis (Coleoptera: Silvanidae) (L. 1758) são pragas secundárias e dependem de que os grãos já estejam danificados para se alimentarem. De acordo com Jian et al. (2012) O. surinamensis apresenta uma distribuição homogênea em grãos armazenados. Dados sobre L. minutus são escassos na literatura.

Os grãos armazenados estão sujeitos a deteriorações e perdas em virtude das interações entre os fenômenos físicos, químicos e biológicos. A falta de uma boa estrutura para infestação, umidade inadequada, porcentagem de impureza junto ao grão no momento do armazenamento, ação de pragas (Santos, 2006; Herrmann et al., 2009) e temperatura em torno de $30^{\circ} \mathrm{C}$ (Fields, 1992, Hagstrum \& Subramanyam, 2006) estão entre as principais causas da deterioração dos grãos durante a armazenagem.

Enquanto os danos causados no campo podem ser compensados pela recuperação da planta danificada ou pelo aumento de produtividade de plantas não atacadas, os prejuízos em grãos armazenados são irrecuperáveis (Fontes et al., 2003). Desta forma, o que preocupa é saber quais as melhores condições para manter armazenados esses grãos contribuindo para sua melhor preservação e menor uso de inseticidas.

Com base neste enfoque, o objetivo do trabalho foi avaliar os danos causados em grãos de arroz em diferentes condições de armazenamento e coexistência de pragas primárias e secundárias.

\section{Material e Métodos}

Os experimentos foram conduzidos no Laboratório de Entomologia da Universidade de Cruz Alta - UNICRUZ em Cruz Alta, RS. A criação foi iniciada com insetos adultos oriundos de silos da região. Foram testadas populações de $S$. zeamais, $O$. surinamensis e L. minutus, insetos praga mantidos em coexistência durante período de criação. Os experimentos ocorreram entre 2009 e 2011 e foram testados os seguintes conjuntos populacionais: $S$. zeamais isolado; $O$. surinamensis isolado; L. minutus isolado; S. zeamais $+O$. surinamensis e $S$. zeamais + L. minutus.

Em potes plásticos com 20 grãos de arroz cada conjunto populacional foi mantido em condições ambientais controladas de temperatura e fotoperíodo $\left(20,25\right.$ e $30^{\circ} \mathrm{C}, 14 \mathrm{~h}$ de fotofase $)$ em estufa tipo B. O. D. (Biological Oxygen Demand) para cinco diferentes densidades de infestação (DI): 10, 20, 40, 80 e 120 indivíduos. Para os conjuntos populacionais $S$. zeamais $+O$. surinamensis e $S$. zeamais $+L$. minutus, cada espécie representou $50 \%$ da DI. Todos os testes experimentais ocorreram em triplicata.

Inicialmente, pesaram-se os grãos de arroz e depois se acondicionaram os espécimes, de acordo com as DI e temperaturas estabelecidas. Após 7 e 14 dias do início dos experimentos os grãos foram separados dos insetos praga para serem novamente pesados. Foram pesados apenas grãos de arroz intactos ou com predação mínima (inferior a $5 \%$ de dano).

Como controle, potes com grãos de arroz sem presença de insetos foram utilizados a fim de determinar as variações de peso pela umidade e corrigir valores de pesagem dos demais tratamentos. Quanto às pesagens, foi utilizada balança digital Deiver instrument m-22 D (capacidade de $2.000 \mathrm{~g}$ e sensibilidade de $0,0001 \mathrm{~g}$ ).

Para os testes estatísticos os dados foram submetidos ao teste de Levene para verificar a homogeneidade das variâncias. As perdas de grãos de arroz por danos dos insetos foram submetidas à análise de variância de dois fatores (DI X temperatura), seguida de teste de Tukey, com $\mathrm{P}<0,05$, através do programa Statistica.

\section{Resultados E Discussão}

As perdas de grãos de arroz variaram de acordo com insetos, DI e temperatura. Para $O$. surinamensis e L. minutus quando isolados os mesmos não geraram danos aos grãos de arroz armazenados, independente da temperatura e DI.

A Figura 1 apresenta os danos ocasionados aos grãos de arroz em diferentes DI e nas temperaturas 20,25 e $30^{\circ} \mathrm{C}$, respectivamente, para $S$. zeamais isolado (A e B), S. zeamais $+O$. surinamensis $(\mathrm{C}$ e D) e $S$. zeamais + L. minutus $(\mathrm{E}$ e F).

Perdas de $100 \%$ ocorreram após 14 dias de infestação na DI 80 indivíduos em $30{ }^{\circ} \mathrm{C}$ para $S$. zeamais + L. minutus (Figura $1 \mathrm{~F})$ e na DI 120 indivíduos tanto em $25^{\circ} \mathrm{C}$, quanto em $30^{\circ} \mathrm{C}$ para S. zeamais isolado (Figura 1B) e S. zeamais + L. minutus (Figura $1 \mathrm{~F}$ ).

Considerando S. zeamais isolado, o aumento da DI apenas não apresentou diferença significativa para perdas de grãos de arroz em 7 dias e $20^{\circ} \mathrm{C}$ porém em 14 dias para a mesma temperatura a perda de grãos de arroz na DI 120 indivíduos foi significativamente superior às DI 10 e 20 indivíduos.

Em 25 e $30^{\circ} \mathrm{C}$, tanto para 7 quanto para 14 dias, observouse um efeito maior de danos de S. zeamais nas maiores DI, principalmente DI 80 e 120 indivíduos. Em $30^{\circ} \mathrm{C}$ com 14 dias de experimento a DI 40 indivíduos não diferiu das DI 80 e 120 indivíduos, mas (assim como em 7 dias) manteve perdas a grãos de arroz significativamente superiores às DI 10 e 20 indivíduos. 
A.

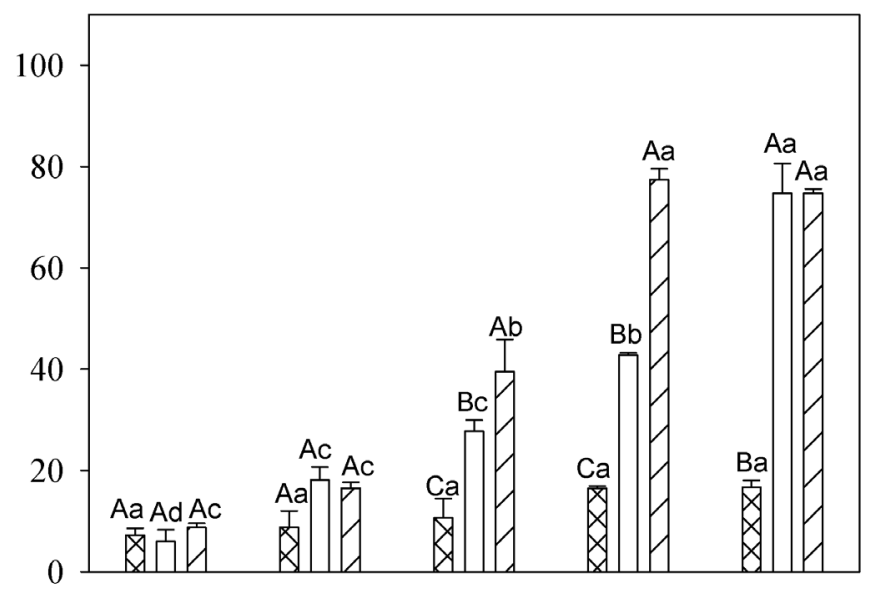

C.

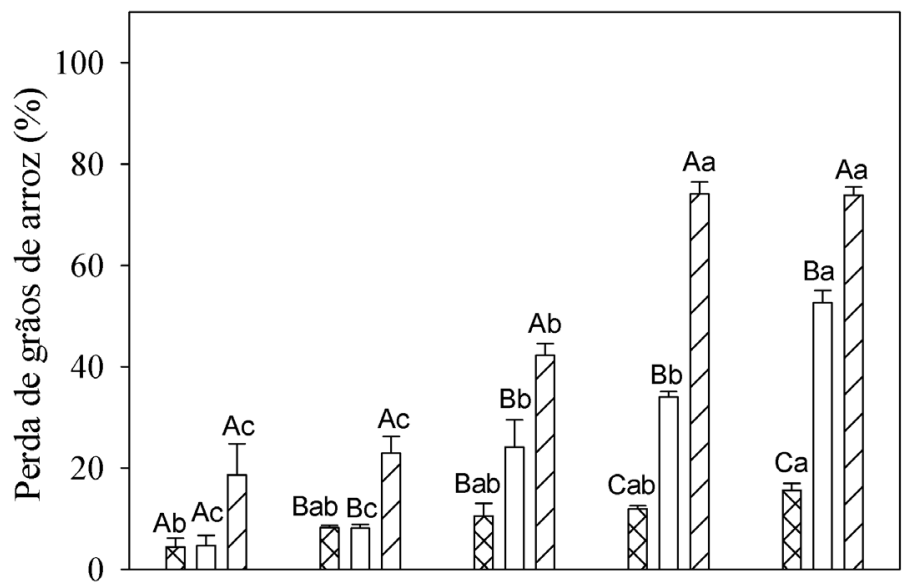

E.

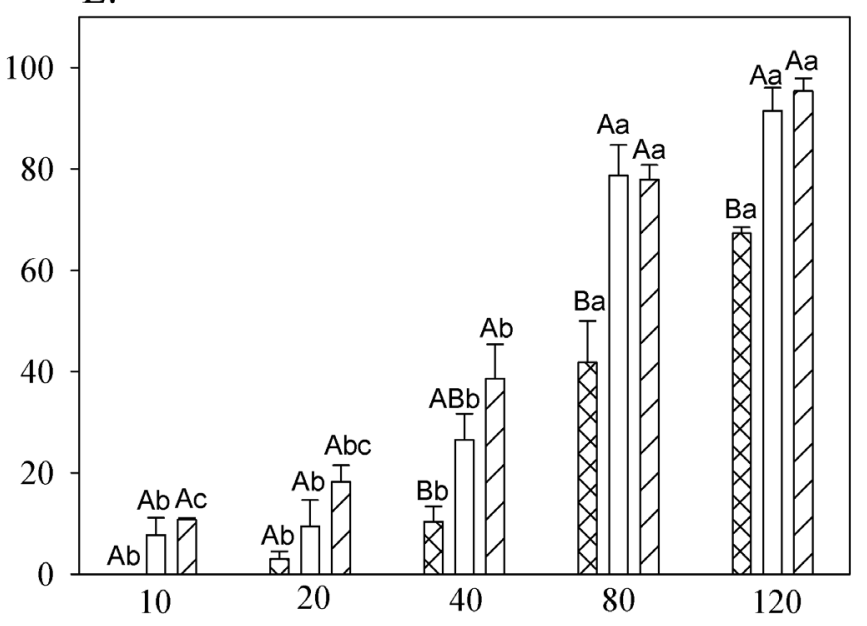

B.

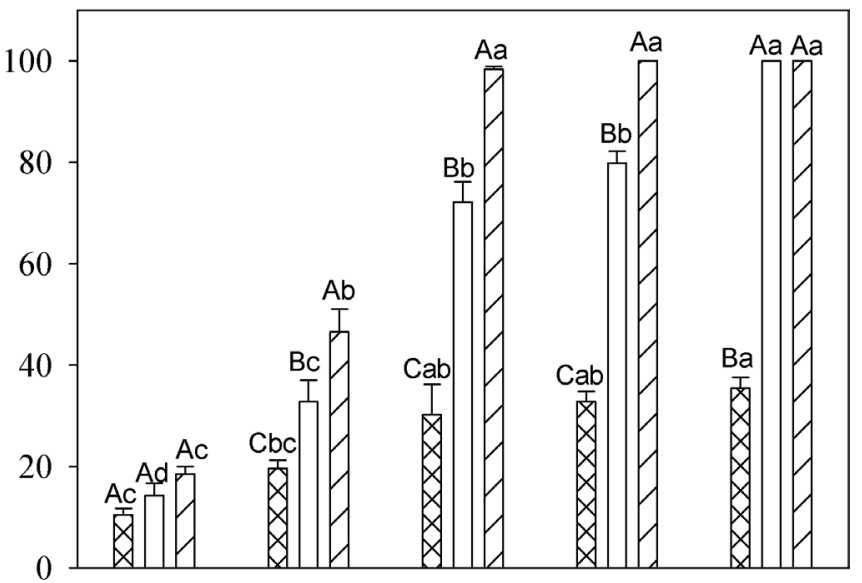

D.

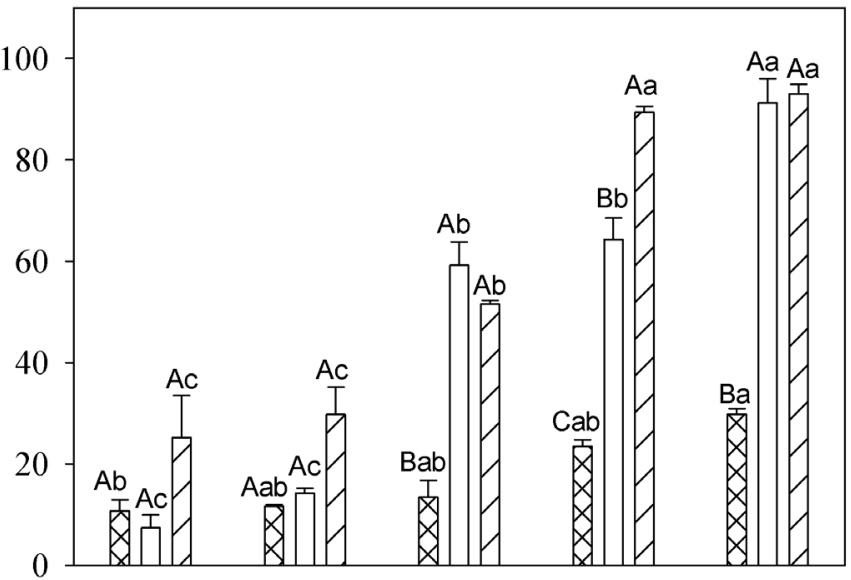

F.

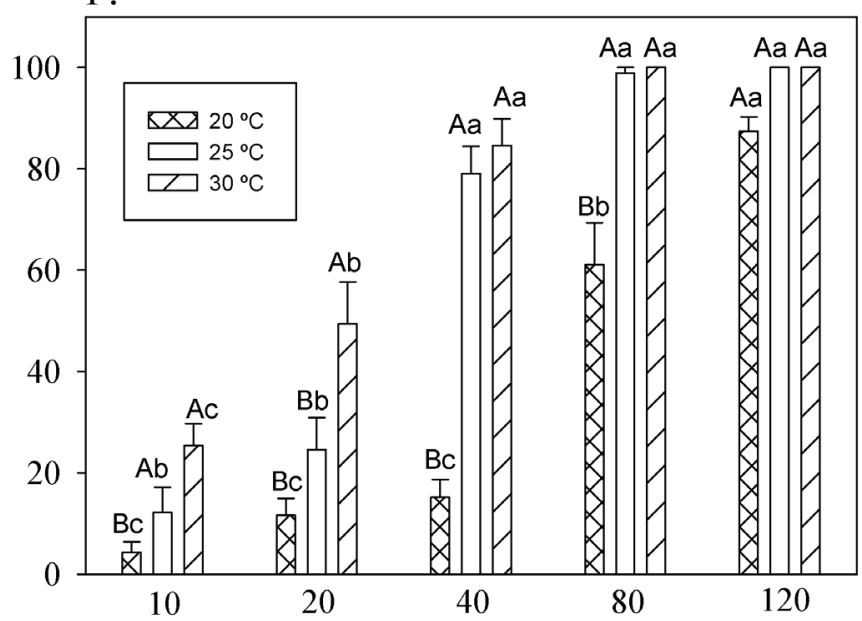

Densidade de estocagem

* Letras maiúsculas referem-se a diferenças entre temperaturas para uma mesma DI. Letras minúsculas referem-se a diferenças entre DI para uma mesma temperatura

Figura 1. Perda de grãos de arroz (\%) por predação de $S$. zeamais isolado após 7 (A) e 14 (B) dias, S. zeamais + O. surinamensis após 7 (C) e 14 (D) dias e S. zeamais + L. minutus após 7 (E) e 14 (F) dias

Com 7 e 14 dias em todas as DI, a exposição na temperatura de $20^{\circ} \mathrm{C}$ reduziu significativamente as perdas de grãos de arroz em comparação com as temperaturas mais elevadas e o aumento de 25 para $30^{\circ} \mathrm{C}$ aumentou significativamente tais perdas para as DI 40 e 80 indivíduos (Figura 1A e B).
Os testes com S. zeamais + O. surinamensis em $20^{\circ} \mathrm{C}$ indicaram que as DI não diferiram significativamente, exceto entre 10 e 120 indivíduos. Em 25 e $30^{\circ} \mathrm{C}$, de maneira geral, as perdas de grãos de arroz foram significativamente maiores nas DI mais altas. 
Tanto para 7 quanto para 14 dias, verificou-se que as menores perdas ocorreram em $20^{\circ} \mathrm{C}$. O aumento de 25 para 30 ${ }^{\circ} \mathrm{C}$ aumentou significativamente as perdas de grãos de arroz em 7 dias para todas as DI (exceto DI 10 indivíduos) e em 14 dias apenas para a DI 80 indivíduos (Figura $1 \mathrm{C}$ e D).

A coexistência $S$. zeamais $+L$. minutus demonstrou que 7 dias após o início dos experimentos as DI 80 e 120 indivíduos registraram perdas a grãos de arroz estatisticamente superiores às demais $\mathrm{DI}$, em todas as temperaturas. $\mathrm{Em} 20^{\circ} \mathrm{C}$, com 14 dias de experimento, verificaram-se perdas significativamente maiores nas DI mais altas.

No mesmo período, em 25 e $30{ }^{\circ} \mathrm{C}$, as DI 40,80 e 120 indivíduos não diferiram entre si mas apresentaram danos significativamente maiores aos grãos de arroz que as demais DI. Em $30^{\circ} \mathrm{C}$ ocorreram perdas significativamente maiores em relação a $20^{\circ} \mathrm{C}$ nas DI 40, 80 e 120 indivíduos com 7 dias e em todas as DI com 14 dias de experimento.

Além disto, a exposição a $25{ }^{\circ} \mathrm{C}$ em relação a $20{ }^{\circ} \mathrm{C}$ demonstrou ocorrer maior efeito de predação aos grãos de arroz em 7 dias nas DI 80 e 120 indivíduos e em 14 dias nas DI 10, 40 e 80 indivíduos (Figura 1E e F).

$\mathrm{O}$ desenvolvimento e o crescimento dos insetos dependem diretamente da temperatura em que eles se encontram. Segundo Fields (1992), a temperatura ótima para o desenvolvimento da maioria das espécies de insetos de produtos armazenados se situa entre $25-33{ }^{\circ} \mathrm{C}$. Hagstrum \& Subramanyam (2006) apontam, por sua vez, que em $30^{\circ} \mathrm{C}$ as populações de insetos de grãos armazenados aumentam rapidamente.

A temperatura ideal para o desenvolvimento de S. zeamais é em torno de $28^{\circ} \mathrm{C}$, com capacidade de apresentar atividade entre 15 e $34^{\circ} \mathrm{C}$ (Dobie et al., 1984). Silva et al. (2006), avaliaram que o crescimento populacional de $S$. zeamais é máximo em $28^{\circ} \mathrm{C}$ e mínimo em $16^{\circ} \mathrm{C}$.

Nakakita \& Ikenaga (1997) e Lazzari et al. (2006) verificaram que quando a temperatura decresce, respectivamente de 30 para $20^{\circ} \mathrm{C}$ e de 26 para $15^{\circ} \mathrm{C}$, a atividade de S. zeamais e Sitophilus oryzae (Coleoptera: Curculionidae) (L. 1763) também diminui. Baldin \& Lara (2004) concluíram que o armazenamento de grãos a $25^{\circ} \mathrm{C}$ favorece o desenvolvimento de Acanthoscelides obtectus (Coleoptera: Bruchidae) (Say 1831) em relação à temperatura de $20^{\circ} \mathrm{C}$, devendo ser evitada nos armazéns.

Em nosso estudo a temperatura de $20{ }^{\circ} \mathrm{C}$ mostrou-se mais eficaz na redução dos danos; acredita-se, portanto, que uma redução maior de temperatura irá contribuir ainda mais com a manutenção da integridade dos grãos de arroz em armazenamento.

Além disto, o tempo de exposição também é fator importante, especialmente na DI 40 indivíduos em 25 e $30^{\circ} \mathrm{C}$, pois com 14 dias após o início dos experimentos os danos não diferiram significativamente das DI 80 e 120 indivíduos para $S$. zeamais isolado e $S$. zeamais + L. minutus (Figura 1). Assim, com um período maior de exposição, uma DI intermediária pode gerar danos similares a DI mais altas.

Antunes et al. (2011) verificaram, ao avaliar os danos causados por populações de $S$. zeamais em grãos de milho híbrido armazenado que quanto maior o tempo de exposição dos grãos maior também é a perda de peso sofrida pelos mesmos.
Smiderle et al. (1997) verificaram que os danos causados a grãos de arroz irrigado por Rhyzopertha dominica (Coleoptera: Brostrycidae) (Fabricius 1972) e Sitophilus sp. são maiores quanto mais longo o período de armazenamento, tanto para as espécies isoladas como em associação.

Para este estudo uma comparação de danos aos grãos de arroz entre $S$. zeamais isolado, $S$. zeamais $+O$. surinamensis e $S$. zeamais $+L$. minutus pode ser vista nas Tabelas 1 e 2, para 7 e 14 dias, respectivamente.

A coexistência $S$. zeamais $+L$. minutus causou danos significativamente superiores aos grãos de arroz que aqueles ocasionados pelas populações de $S$. zeamais isolado e $S$. zeamais

Tabela 1. Perda de grãos de arroz (\%) por $S$. zeamais isolado, $S$. zeamais + O. surinamensis, $S$. zeamais + L. minutus após 7 dias de infestação nas diferentes densidades de infestação (DI) e temperaturas

\begin{tabular}{rrcr}
\hline DI & S. zeamais & $\begin{array}{c}\text { S. zeamais }+ \\
\text { 0. surinamensis }\end{array}$ & $\begin{array}{c}\text { S. zeamais }+ \\
\text { L. minutus }\end{array}$ \\
& & $20^{\circ} \mathrm{C}$ \\
10 & $7,22 \pm 1,38 \mathrm{a}$ & $4,40 \pm 1,72 \mathrm{a}$ & $0,14 \pm 0,08 \mathrm{a}$ \\
20 & $8,80 \pm 3,19 \mathrm{a}$ & $8,18 \pm 0,50 \mathrm{a}$ & $2,98 \pm 1,50 \mathrm{a}$ \\
40 & $10,67 \pm 3,75 \mathrm{a}$ & $10,85 \pm 2,45 \mathrm{a}$ & $10,35 \pm 3,01 \mathrm{a}$ \\
80 & $16,45 \pm 0,42 \mathrm{~b}$ & $11,92 \pm 0,58 \mathrm{~b}$ & $41,81 \pm 8,16 \mathrm{a}$ \\
120 & $16,65 \pm 1,36 \mathrm{~b}$ & $15,58 \pm 1,39 \mathrm{~b}$ & $67,29 \pm 1,21 \mathrm{a}$ \\
\hline \multicolumn{4}{c}{$25^{\circ} \mathrm{C}$} \\
10 & $5,95 \pm 2,36 \mathrm{a}$ & $4,68 \pm 2,01 \mathrm{a}$ & $7,72 \pm 3,43 \mathrm{a}$ \\
20 & $18,10 \pm 2,56 \mathrm{a}$ & $8,08 \pm 0,79 \mathrm{a}$ & $9,46 \pm 5,19 \mathrm{a}$ \\
40 & $27,73 \pm 2,20 \mathrm{a}$ & $24,10 \pm 5,42 \mathrm{a}$ & $26,52 \pm 5,13 \mathrm{a}$ \\
80 & $42,79 \pm 0,47 \mathrm{~b}$ & $34,01 \pm 1,06 \mathrm{~b}$ & $78,72 \pm 6,02 \mathrm{a}$ \\
120 & $74,71 \pm 5,90 \mathrm{~b}$ & $52,63 \pm 2,44 \mathrm{C}$ & $91,48 \pm 4,54 \mathrm{a}$ \\
\hline \multicolumn{4}{c}{$30{ }^{\circ} \mathrm{C}$} \\
10 & $8,72 \pm 0,83 \mathrm{a}$ & $18,66 \pm 6,13 \mathrm{a}$ & $10,76 \pm 0,35 \mathrm{a}$ \\
20 & $16,53 \pm 1,14 \mathrm{a}$ & $22,91 \pm 3,34 \mathrm{a}$ & $18,20 \pm 3,31 \mathrm{a}$ \\
40 & $39,46 \pm 6,40 \mathrm{a}$ & $42,25 \pm 2,31 \mathrm{a}$ & $38,59 \pm 6,81 \mathrm{a}$ \\
80 & $77,39 \pm 2,15 \mathrm{a}$ & $74,10 \pm 2,36 \mathrm{a}$ & $77,94 \pm 2,81 \mathrm{a}$ \\
120 & $74,78 \pm 0,81 \mathrm{~b}$ & $73,87 \pm 1,56 \mathrm{~b}$ & $95,35 \pm 2,56 \mathrm{a}$ \\
\hline
\end{tabular}

* Médias seguidas de mesma letra minúscula na coluna não diferem estatisticamente

Tabela 2. Perda de grãos de arroz (\%) por S. zeamais isolado, S. zeamais + O. surinamensis, S. zeamais + L. minutus após 14 dias de infestação nas diferentes densidades de infestação (DI) e temperaturas

\begin{tabular}{rccc}
\hline DI & S. zeamais & $\begin{array}{c}\text { S. zeamais }+ \\
\text { 0. surinamensis }\end{array}$ & $\begin{array}{c}\text { S. zeamais }+ \\
\text { L. minutus }\end{array}$ \\
& & $20^{\circ} \mathrm{C}$ \\
10 & $10,43 \pm 1,31 \mathrm{a}$ & $10,70 \pm 2,23 \mathrm{a}$ & $4,26 \pm 2,15 \mathrm{a}$ \\
20 & $19,53 \pm 1,72 \mathrm{a}$ & $11,64 \pm 0,37 \mathrm{a}$ & $11,56 \pm 3,41 \mathrm{a}$ \\
40 & $30,19 \pm 5,98 \mathrm{a}$ & $13,39 \pm 3,38 \mathrm{a}$ & $15,13 \pm 3,57 \mathrm{a}$ \\
80 & $32,82 \pm 1,98 \mathrm{~b}$ & $23,42 \pm 1,33 \mathrm{~b}$ & $61,02 \pm 8,32 \mathrm{a}$ \\
120 & $35,43 \pm 2,13 \mathrm{~b}$ & $29,80 \pm 1,12 \mathrm{~b}$ & $87,36 \pm 2,88 \mathrm{a}$ \\
\hline \multicolumn{4}{c}{$25^{\circ} \mathrm{C}$} \\
10 & $14,26 \pm 2,33 \mathrm{a}$ & $7,37 \pm 2,55 \mathrm{a}$ & $12,14 \pm 4,98 \mathrm{a}$ \\
20 & $32,76 \pm 4,25 \mathrm{a}$ & $14,23 \pm 0,97 \mathrm{~b}$ & $24,57 \pm 6,28 \mathrm{ab}$ \\
40 & $72,15 \pm 3,99 \mathrm{ab}$ & $59,18 \pm 4,67 \mathrm{~b}$ & $79,04 \pm 5,34 \mathrm{a}$ \\
80 & $79,85 \pm 2,41 \mathrm{~b}$ & $64,32 \pm 4,22 \mathrm{~b}$ & $98,85 \pm 1,15 \mathrm{a}$ \\
120 & $100,00 \pm 0,00 \mathrm{a}$ & $91,25 \pm 4,74 \mathrm{a}$ & $100,00 \pm 0,00 \mathrm{a}$ \\
\hline \multicolumn{4}{c}{$30{ }^{\circ} \mathrm{C}$} \\
10 & $18,49 \pm 1,51 \mathrm{a}$ & $25,16 \pm 8,36 \mathrm{a}$ & $25,35 \pm 4,36 \mathrm{a}$ \\
20 & $46,51 \pm 4,50 \mathrm{ab}$ & $29,73 \pm 5,44 \mathrm{~b}$ & $49,39 \pm 8,24 \mathrm{a}$ \\
40 & $98,32 \pm 0,57 \mathrm{a}$ & $51,51 \pm 0,78 \mathrm{C}$ & $84,58 \pm 5,26 \mathrm{~b}$ \\
80 & $100,00 \pm 0,00 \mathrm{a}$ & $89,36 \pm 1,19 \mathrm{~b}$ & $100,00 \pm 0,00 \mathrm{a}$ \\
120 & $100,00 \pm 0,00 \mathrm{a}$ & $93,01 \pm 1,89 \mathrm{a}$ & $100,00 \pm 0,00 \mathrm{a}$ \\
\hline
\end{tabular}

* Médias seguidas de mesma letra minúscula na coluna não diferem estatisticamente 
+ O. surinamensis nas DI 80 e 120 indivíduos para todas as temperaturas (exceto DI 80 indivíduos em $30^{\circ} \mathrm{C}$ ) com 7 dias após o início dos experimentos (Tabela 1) mas com 14 dias isto foi verificado apenas em $20^{\circ} \mathrm{C}$ (DI 80 e 120 indivíduos) e em $25^{\circ} \mathrm{C}$ (DI 80 indivíduos) (Tabela 2).

Com 14 dias de experimento em $30^{\circ} \mathrm{C}$, a interação $S$. zeamais $+L$. minutus ocasionou, de maneira geral, danos significativamente maiores aos grãos de arroz que $S$. zeamais $+O$. surinamensis (Tabela 2).

Quando S. zeamais estava isolado as perdas foram significativamente superiores que $S$. zeamais $+O$. surinamensis com 7 dias de experimento em $20^{\circ} \mathrm{C}$ e DI 120 indivíduos (Tabela 1) e com 14 dias de experimento em $25^{\circ} \mathrm{C}$ e DI 20 indivíduos e em $30^{\circ} \mathrm{C}$ e DI 40 e 80 indivíduos. Adicionalmente, S. zeamais isolado causou perdas aos grãos de arroz significativamente maiores que $S$. zeamais $+L$. minutus apenas em $30^{\circ} \mathrm{C}$ e DI 40 indivíduos (Tabela 2).

As Tabelas 1 e 2 demonstram que nas maiores DI a associação de $S$. zeamais é mais danosa aos grãos de arroz quando em associação com L. minutus do que quando em associação com $O$. surinamensis.

Apesar da grande preocupação no controle de pragas primárias, este trabalho demonstra que pelo menos nas situações testadas, L. minutus é uma praga secundária que merece grande atenção de vez que sua ação danosa, quando em associação com S. zeamais, não foi inferior significativamente a $S$. zeamais isolado na maioria dos tratamentos testados nas mesmas condições.

Por outro lado, $O$. surinamensis $+S$. zeamais foi a associação menos danosa aos grãos de arroz evidenciando que as pragas secundárias se comportaram de maneira distinta na presença da praga primária, em que $L$. minutus apresentou um potencial de dano maior que $O$. surinamensis. Experimentos realizados por Pinto et al. (2002) demonstraram que grãos de trigo expostos a DI maiores de $S$. zeamais perderam a qualidade nutricional, o que é similar ao observado neste estudo, cujas maiores DI para S. zeamais (isolado ou em coexistência) geraram perdas quantitativas que inviabilizam os grãos de arroz para o consumo.

Silva et al. (2003) observaram, ao analisar as perdas causadas por $S$. zeamais e $R$. dominica a grãos de trigo em diferentes DI, que ambas as espécies (isoladas) causaram mais danos quando os grãos foram expostos às maiores DI, o que também foi observado no presente estudo e não apenas para $S$. zeamais isolado mas também para $S$. zeamais em coexistência com os insetos-praga secundários.

Em trabalho realizado por Alencar et al. (2011) em que os grãos de milho foram expostos à ação de populações de $S$. zemais e Tribolium castaneum (Coleoptera: Tenebrionidae) (Herbst, 1797), isolados e em associação, a infestação dos grãos com $S$. zeamais isolado causou perda significativa da qualidade dos grãos porém o tratamento de coexistência causou danos ainda maiores.

No presente estudo a coexistência $S$. zeamais $+O$. surinamensis não causou danos superiores àqueles registrados para $S$. zeamais isolado mas a coexistência $S$. zeamais $+L$. minutus ocasionou danos superiores que os $S$. zeamais isolado nas maiores DI.

\section{Conclusões}

1. Apenas os testes com S. zeamais isolado ou em coexistência, acarretaram danos aos grãos de arroz armazenados cuja interação $S$. zeamais + L. minutus registrou as maiores perdas.

2. Os maiores efeitos de danos aos grãos de arroz armazenados ocorreram nas maiores DI, especialmente nas temperaturas 25 e $30^{\circ} \mathrm{C}$, as quais não são recomendadas para $\mathrm{o}$ armazenamento de grãos.

3. Uma DI intermediária em um tempo maior de exposição acarreta prejuízos similares a DI mais altas.

4. O armazenamento correto em temperaturas mais baixas associado ao monitoramento e controle de infestação não apenas de pragas primárias mas também de pragas secundárias, é sugerido como medida efetiva para redução de danos a grãos de arroz armazenados.

\section{Agradecimentos}

Os autores agradecem a Marcelo Lopes da Silva pelo auxílio no delineamento experimental. Roberta K. Marcon agradece a bolsa PIBIC-Unicruz.

\section{Literatura Citada}

Alencar, E. R.; Faroni, L. R. D.; Ferreira, L. G.; Costa, A. R. da; Pimentel, M. A. G. Qualidade de milho armazenado e infestado por Sitophilus zeamais e Tribolium castaneum. Engenharia na Agricultura, v.19, p.9-18, 2011.

Antunes, L. E. G.; Viebrantz, P. C.; Gottardi, R.; Dionello, R. G. Características físico-químicas de grãos de milho atacados por Sitophilus zeamais durante o armazenamento. Revista Brasileira de Engenharia Agrícola Ambiental, v.15, p.615-620, 2011.

Baldin, E. L. L.; Lara, M. F. Efeito de temperaturas de armazenamento e de genótipos de feijoeiro sobre a resistência a Acanthoscelides. Neotropical Entomology, v.33, p.365-369, 2004.

Copatti, C. E.; Gasparetto, F. M. Diversidade de insetos em diferentes tipos de bordaem um fragmento de Floresta Ombrófila Mista. Revista Biociências, v.18, p.32-40, 2012.

Copatti, E. C.; Garcia, L. O.; Baldisserotto, B. Uma importante revisão sobre o impacto de agroquímicos da cultura de arroz em peixes. Biodiversidade Neotropica, v.9, p.235-242, 2009.

Dobie, P.; Haines, C. P.; Hodges, R. J.; Prevett, P. F. Insects and arachnids of tropical stored products, their biology and identification: A training manual. London: Tropical Development and Research Institute, 1984. 131p.

Fields, P. G. The control of stored-product insects with extreme temperatures. Journal of Stored Products Research, v.28, p.89-118 1992.

Fontes, L. S.; Almeida Filho, A. J.; Arthur, V. Danos causados por Sitophilus oryzae (Linné, 1763) e Sitophilus zeamais Motschulsky, 1855 (Coleoptera: Curculionidae) em cultivares de arroz (Oryza sativa L.). Arquivos do Instituto Biológico, v.70, p.303-307, 2003.

Hagstrum, D. W.; Subramanyam, B. Fundamentals of storedproduct entomology. St. Paul: American Association of Cereal Chemists, 2006. 300p. 
Herrmann, D. R.; Carvalho, M. J. de; Zachow, K.; Scholz, F.; Rabbers, D.; Tsutsumi, C. Y.; Zonin, W. Avaliação da resistência de cultivares de milho ao ataque de Sitophilus sp. em grãos armazenados. Revista Brasileira de Agroecologia, v.4, p.4290-4293, 2009.

Jian, F.; Larson, R.; Jayas, D. S.; White, N. D. G. Three dimensional temporal and spatial distribution of adult Oryza ephilus surinamensis and Sitophilus oryzae in stored wheat under different temperatures, moisture contents, and adult densities. Journal of Stored Products Research, v.49, p.155-165, 2012.

Lazzari, S. M. N.; Karkle, A. F.; Lazzari, F. A. Resfriamento artificial para o controle de Coleoptera em arroz armazenado em silo metálico. Revista Brasileira de Entomologia, v.50, p.293-296, 2006.

Nakakita, H.; Ikenaga, H. Action of low temperature on physiology of Sitophilus zeamais Motschulsky and Sitophilus oryzae (L.) (Coleoptera: Curculionidae) in rice storage. Journal of Stored Products Research, v.33, p.31-38, 1997.

Pinto, U. M.; Faroni, L. R. D.; Alves, W. M.; Silva, A. A. L. da. Influência da densidade populacional de Sitophilus zeamais (Motsch.) sobre a qualidade do trigo destinado à panificação. Acta Scientiarum, v.24, p.1407-1412, 2002.

Ribeiro, C. S. N.; Martins, G. V.; Guimarães, J. F. R.; Silva, E. F. Resistência de genótipos de arroz a pragas de grãos armazenados. Revista Caatinga, v.25, p.183-187, 2012.
Santos, J. P. Controle de pragas durante o armazenamento de milho. Lagoas: Embrapa Milho e Sorgo, 2006. 7p. Circular Técnica, n.84.

Silva, A. A. L. da; Faroni, L. R. D. A.; Guedes, R. N. C.; Martins, J. H.; Pimentel, M. A. G. Modelagem das perdas causadas por Sitophilus zeamais e Rhyzopertha dominica em trigo armazenado. Revista Brasileira Engenharia Agrícola Ambiental, v.7, p.292-296, 2003.

Silva, A. A. L. da; Faroni, L. R. D. A.; Guedes, R. N. C.; Martins, J. H.; Pimentel, M. A. G. Modelos analíticos do crescimento populacional de Sitophilus zeamais em trigo armazenado. Revista Brasileira de Engenharia Agrícola e Ambiental, v.10, p.155-161, 2006.

Silveira, R. D.; Faroni, L. R. D. A.; Pimental, M. A. G.; Zocolo, G. J. Influência da temperatura do grão de milho, no momento da pulverização, e do período de armazenamento, na mortalidade de Sitophilus zeamais e Tribolium castaneum, pela mistura bifenthrin e pirimifós-metil. Revista Brasileira de Armazenamento, v.31, p.120-124, 2006.

Smiderle, O. J.; Santos Filho, B. G. dos; Santos, D. S. B. dos; Loeck, A. E.; Silva, J. B. da. Qualidade física e fisiológica de sementes de arroz irrigado (Oryza sativa L.) submetidas ao ataque de Rhizopertha dominica Fabricius e Sitophilus sp. durante o armazenamento. Revista Brasileira de Sementes, v.19, p.1-8, 1997. 